\title{
Using standardised data as a means to overcome the limitations of fitting polynomials in a genetic analysis of lifetime ewe weights using random regression
}

G E Pollott, G Galea

Royal Veterinary College, London, United Kingdom

Email:gpollott@rvc.ac.uk

Introduction It is commonly thought that by selecting for increased early lamb growth the mature weight of the breeding ewe population will increase. This notion has rarely been tested using measurements taken on the same animals since most meat animals are slaughtered at a young age and most breeding sheep are rarely weighed throughout their lives. Random regression is commonly used to study the genetics of a trait repeatedly measured on a linear scale e.g. time. In such analyses an overall curve is used to 'correct' the data for variation in the trait on the linear scale and random regression curves are fitted for each animal to investigate how additive variance changes along this scale. Polynomials are commonly used for both regressions (fixed and random) but such methods suffer from drawbacks. Firstly there may be heterogeneous variance over the linear scale and a polynomial may not best describe the overall trend in the trait. In this research the use of standardised data was investigated as a means to circumvent both problems in an analysis of lifetime liveweight in ewes and the results used to investigate the genetics of lifetime ewe weights.

Materials and methods The data used in this study came from a fully recorded and pedigreed flock of 600 ewes weighed three-times a year (mating, lambing and weaning) and also as lambs at birth, 6 and 16-weeks of age. Data were available for 1,390 ewes born over a 13-year period. Research aimed at finding an appropriate model to describe lifetime ewe weight (Pollott and Galea, 2010) highlighted the lack of fit for the commonly used polynomial approach and no suitable linear model was found to use in a random regression. In addition the variance of liveweight was heterogeneous over the 7-year lifespan of the ewes and using (potentially) 21 different variance components was not an option due to program limitations (Gilmour et al, 2006). To overcome both problems the data was standardised for each of the 21 weights after Pollott and Greeff (2004). Standardisation was achieved by calculating the mean and SD for each of the 21 weighing times. Each individual ewe weight was then 'standardised' by subtracting the appropriate mean and dividing by the appropriate SD. The effect of this was to give each weight a similar mean and variance. The standardised data were analysed using a mixed model in ASREML (Gilmour et al., 2006) fitting birth type, birth year and dam age as fixed effects, and genetic and maternal random regression terms for ewe and dam respectively. Comparative results were calculated using a univariate animal model at each weight, and by setting ASREML to calculate a different residual variance for 10 of the most variable weights. Heritability values were calculated as the ratio of the ewe variance to the phenotypic variance, where the phenotypic variance was the sum of the ewe and residual variances or the sum of the ewe, residual and dam variances as appropriate. Standard errors were calculated as described in Gilmour et al. (2006). The covariance functions derived from these analyses were used to investigate the change in genetic correlation between young and older ages.

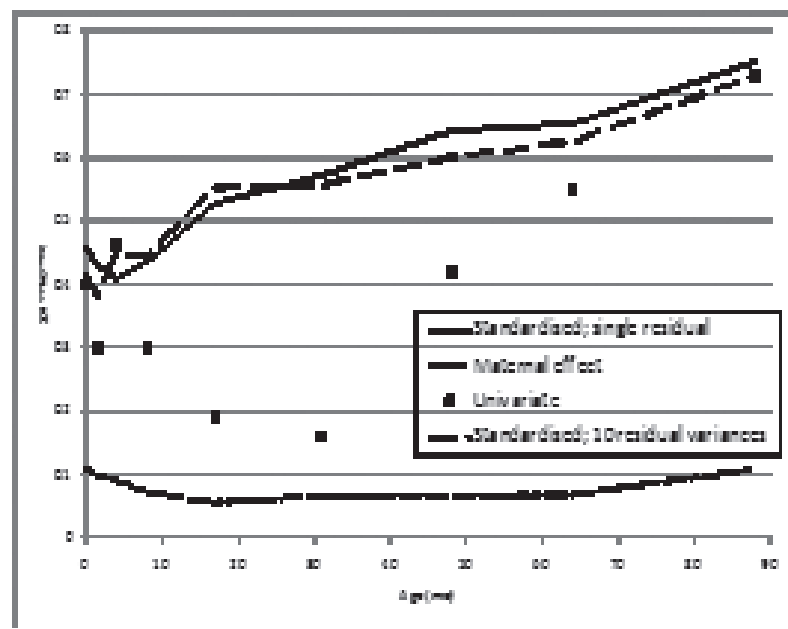

Results The results of fitting the various models are summarised in the Figure as heritability values. The general trend in heritabilities was to increase with age but some differences were found in early life. When fitting a single residual and the maternal effect, 6-week weight heritability was lower than that at birth. The univariate analyses showed a marked fall in heritability after first mating but rose again after about 3 years of age. Both standardised random regression analyses showed a similar pattern of heritability and the change in residual variance with age made little difference to the results and were greater than the corresponding univariate estimate. The genetic correlations shown in Table 1 show a very low level of association between early lamb weights and a sample of weights from the same animals as mature ewes. Even at 4 months of age, lambs only had a genetic correlation of 0.24 with weight at 2 years; at older ages this correlation was reduced.

Figure 1 The heritability of liveweight calculated by 3 different methods.

Table 1 The genetic correlation between lamb weights and the mature weight of the ewe.

\begin{tabular}{llllll}
\hline \hline & Mating 2 & Lambing 2 & Lambing 4 & Lambing 6 & Mating 7 \\
\hline Birth & 0.11 & 0.10 & 0.11 & 0.06 & 0.04 \\
6 weeks & 0.16 & 0.14 & 0.12 & 0.09 & 0.06 \\
16 weeks & 0.24 & 0.21 & 0.14 & 0.13 & 0.10 \\
\hline \hline
\end{tabular}

Conclusions Using standardised data is a suitable method for overcoming a poorly fitting overall regression model in random regression analyses. Using this method it was apparent that early lamb weight was not correlated with mature ewe weight to any great degree.

References

Gilmour, A. R., Gogel, B. J., Cullis, B. R. and Thompson, R. 2006. Asreml User Guide Release 2.0.

Pollott, G. E. and Galea, G. 2010. Proceedings of the British Society of Animal Science, 126.

Pollott, G. E. and Greeff, J. C. 2004. Journal of Animal Science 82:2840-2851. 